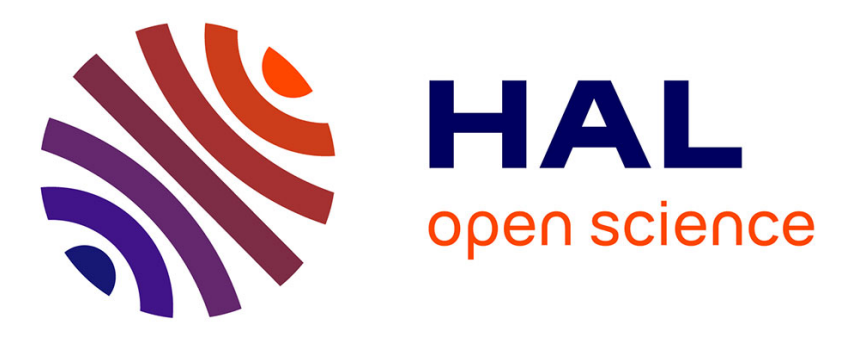

\title{
The Berlin initiative study: the methodology of exploring kidney function in the elderly by combining a longitudinal and cross-sectional approach
}

Elke S. Schaeffner, Markus Giet, Jens Gaedeke, Markus Tölle, Natalie Ebert, Martin K. Kuhlmann, Peter Martus

\section{To cite this version:}

Elke S. Schaeffner, Markus Giet, Jens Gaedeke, Markus Tölle, Natalie Ebert, et al.. The Berlin initiative study: the methodology of exploring kidney function in the elderly by combining a longitudinal and cross-sectional approach. European Journal of Epidemiology, 2010, 25 (3), pp.203-210. 10.1007/s10654-010-9424-x . hal-00568379

\section{HAL Id: hal-00568379 \\ https://hal.science/hal-00568379}

Submitted on 23 Feb 2011

HAL is a multi-disciplinary open access archive for the deposit and dissemination of scientific research documents, whether they are published or not. The documents may come from teaching and research institutions in France or abroad, or from public or private research centers.
L'archive ouverte pluridisciplinaire HAL, est destinée au dépôt et à la diffusion de documents scientifiques de niveau recherche, publiés ou non, émanant des établissements d'enseignement et de recherche français ou étrangers, des laboratoires publics ou privés. 
The Berlin Initiative Study (BIS):

The methodology of exploring kidney function in the elderly by combining a longitudinal and cross-sectional approach

\author{
Elke S. Schaeffner \\ Markus van der Giet \\ Jens Gaedeke \\ Markus Tölle \\ Natalie Ebert \\ Martin K. Kuhlmann \\ Peter Martus
}

\begin{abstract}
${ }^{1}$ Division of Nephrology, Charité University Medicine, Campus Virchow, Berlin,
${ }^{2}$ Charité Campus Benjamin Franklin, ${ }^{4}$ Charité Campus Mitte,${ }^{3}$ Vivantes Klinikum am

Friedrichshain, Department of Nephrology (M.K), ${ }^{5}$ Institute for Biostatistics and

Clinical Epidemiology, Charité, Berlin, Germany (P.M.)
\end{abstract}

Corresponding Address:

PD Dr. Elke Schaeffner, MS

Charité Hospital, Campus Virchow Klinikum

Division of Nephrology and Intensive Care Medicine

Augustenburger Platz 1

13353 Berlin; Germany

Phone: +49 (0)30 450665517

Fax: +49 (0)30 450553909

Email: elke.schaeffner@charite.de 
Word count: Abstract: 256, Text: 3966

Running title: Exploring kidney function in the elderly 


\section{ABSTRACT}

Epidemiologic data on incidence, prevalence and risk factors for chronic kidney disease (CKD) and its progression to kidney failure in people $\geq 70$ years is scarce. This lack may have two reasons: First, the issue has only recently gained importance by the changing demographics characterized by an aging society. Secondly, a validated method for estimating kidney function in terms of glomerular filtration rate (GFR) in the elderly is still lacking.

In this paper we describe the methodology of a combined longitudinal and crosssectional approach of a population based study which will start in January 2010. The aims of the study are to identify prevalent and incident cases of CKD as well as comorbidities and associated risk factors for progression of disease in this specific agegroup. To assess prevalence, a new GFR estimation equation is to be developed. In a longitudinal approach a population based, age stratified sample of 2000 subjects $\geq 70$ years will be randomly drawn from a data base of a large health insurance company. Interview, physical examination, and preliminary estimation of GFR, based on serum creatinine will be performed. The entire cohort will be followed over the course of two years.

In a cross-sectional approach a subsample of 600 subjects will be defined based on preliminary GFR values. Kidney function will be determined by measuring plasma clearance of an exogenous filtration marker (Iohexol). A new GFR-equation will be developed and validated using lohexol clearance as gold standard to estimate GFR accurately and precisely. Data of 2000 subjects will be used to estimate prevalence of CKD.

Key words: CKD, design, epidemiological study, formula, glomerular filtration rate, incidence, older persons, prevalence 


\section{INTRODUCTION}

There is an ongoing demographic shift in Germany that is characterized by less children and a growing number of elderly people with higher life expectancy. Whereas in 2005 there were almost as many newborns as 60 year olds, in 2050 Germany expects double the number of 60 year olds compared to newborns (1). Chronic kidney disease (CKD) is a large and growing problem among aging populations. A number of studies show an age-dependent loss of kidney function after 30 years of age. The process of renal aging is complex and includes anatomical, structural as well as functional changes in the kidneys (2). It is well known that the number of glomeruli decreases with age (3). The cause of age-related increases in renal fibrosis leading to glomerulosclerosis, interstitial fibrosis, tubular atrophy, vascular sclerosis, and consequently to loss of renal function are poorly understood; however, in animal models, collagen seems to accumulate with age in the glomerulus, peritubular capillary, and tubulointerstitium (4). Telomere shortening, implicated in somatic cellular senescence pathways, is observed in aging human kidneys (5) to mention just a few possible mechanisms of decline. However, not everyone seems to follow that pattern, some even do not show any loss of kidney function but rather an increase as could be shown in a large longitudinal study (6). It is unclear if the loss in kidney function is the result of immunologic, infectious, toxic or ischemic influences or is associated with the observed hyperfiltration resulting in glomerulosclerosis.

This unclarity about renal aging does not only refer to causes, co-morbidities, or associated risk factors. Already the assessment of kidney function in people $\geq 70$ years of age is problematic: In general glomerular filtration rate (GFR) is considered the best overall index of kidney function in health and disease. Since it cannot be 
measured easily in clinical practice it is nowadays estimated from equations on the basis of serum-creatinine, age, gender, race and body size (7). One such formula, the Modification of Diet in Renal Disease (MDRD)-equation has gained worldwide acceptance. One major limitation is that in people over 70 years of age the equation is not validated and clearly underestimates kidney function. Even though more accurate than the Cockcroft-Gault formula, an older and also widely used equation (8) this underestimation could also be demonstrated for healthy individuals (9),(10),(11). A very recently developed formula, the CKD-Epi equation, is supposed to be more precise and accurate than the MDRD equation. Again, elderly people were underrepresented in this study (12).

Elderly persons are generally underrepresented in studies about estimation equations (13). In addition, stages of CKD developed by National Kidney Foundation's "kidney disease outcomes quality initiative" (KDOQI) do not take into account age related loss of kidney function (14). For an 80 year old woman with a serum-creatinine of $1.2 \mathrm{mg} / \mathrm{dL}$ the MDRD equation calculates a GFR of only 45 $\mathrm{ml} / \mathrm{min} / 1.73 \mathrm{~m}^{2}$. Therefore the patient will be classified as kidney disease, stage $3 . \mathrm{In}$ reality this serum-creatinine of $1.2 \mathrm{mg} / \mathrm{dL}$ may correspond to an age-adapted normal kidney function without any evidence of kidney disease. None of the huge previous population based studies regarding prevalence of chronic kidney disease have used a validated equation for GFR-estimation in people $\geq 70$ years of age (15). One of the few existing studies analysing cardiovascular risk factors and kidney function in the elderly is based on serum creatinine levels only (16). The interpretation of a calculated MDRD-GFR is difficult without reference values. A current analysis compared GFR of persons with risk factors such as hypertension, diabetes, and cardiovascular disease with the GFR of a "reference-population" without such risk factors (17). The GFR was calculated with the MDRD equation categorizing patients 
between the age of 18 and 85 . Results showed that the KDOQI-staging is not suited for women $>50$ and men $>60$ years of age.

Therefore epidemiologic data on normal as well as reduced kidney function in people $\geq 70$ years of age are highly needed in order to estimate GFR not only on the basis of serum creatinine. Without this prerequisite neither assumptions regarding prevalence, progression and risk factors of CKD can be made nor can preventive or therapeutic strategies be developed.

\section{MATERIALS AND METHODS}

\section{Design overview}

We describe design and methods of the "Berlin Initiative Study", a population based cohort study that started its pilot in November 2009. The main study will start January 2010 and will presumably end in 2014 . The study follows a two part design: First there is a longitudinal prospective cohort study following the participants over a prespecified period of time seeing them twice for a baseline- and a follow-up visit (figure 1). The longitudinal approach aims to assess incidence of CKD, risk factors for development and progression of disease as well as associated co-morbidities.

Secondly, there will be a cross-sectional design aiming to develop a new estimating equation to estimate GFR more accurately and precisely and thus assess prevalence of CKD in the population of elderly people $\geq 70$ years of age.

The study was approved by the local ethics committee.

\section{Aims and Hypotheses}

Longitudinal design 
1. To assess progression of CKD in the elderly and the natural course of kidney function in this population

2. To assess the incidence of clinically relevant decrease in GFR ( $>3 \mathrm{ml} / \mathrm{min} / \mathrm{year}$ ) applying the classical MDRD equation and the recently published CKD-Epi equation and/or requirement of dialysis within the follow-up of 2 years

3. To assess the incidence of cardiovascular events and mortality.

4. To assess associations between risk factors and kidney function / requirement of dialysis and between kidney function and cardiovascular events

Cardiovascular events are defined as non fatal or fatal myocardial infarction or cardiac death; non fatal or fatal stroke (ischemic/hemorrhagic); revascularisation, including angioplasty or bypass of the coronary arteries or other arteries, and non traumatizing limb amputation.

Cross-sectional design

1. To develop a new equation to predict GFR in a subset of the selected study population (development or learning cohort)

2. To validate the new equation in another subset of the selected study population (validation cohort)

3. To assess the prevalence of CKD in people $\geq 70$ years of age applying the classical MDRD equation and the by then newly developed Berlin Initiative Study (BIS) equation

4. To assess risk factors that correlate with prevalence of CKD

\section{Longitudinal design}


It is planned that 2000 participants will be seen for a baseline visit at one of 13 study sites across Berlin. This baseline visit includes a computer-based standardised questionnaire including questions regarding demographics, lifestyle variables, comorbidities, emphasizing cardiovascular events, and medication. There will be a short examination including blood pressure (identical electronic blood pressure meter available at every study site), heart rate, body mass index, and waist-to-hip-ratio measurement. All measurements are carried out according to pre-specified standard operating procedures. Blood and urine samples will be taken and GFR will be estimated using the MDRD-and the new CKD-Epi equation. The urine samples include testing for (micro)albumin and creatinine as this is part of the current KDOQI classification of CKD and since it was shown that proteinuria in the elderly is predicting progression in kidney function decline (18). Additional to an instant laboratory analysis, storage of blood and urine samples at $-80^{\circ} \mathrm{C}$ will be done for further analyses including genetics. For genetics we use DNA from the white bood cell fraction to perform comprehensive SNP analyses in order to look for gene polymorphysms that might determine renal function in the elderly or correlate with factors influencing renal function in the elderly.

After two years these 2000 participants are planned to be seen again (follow-up visit) for an identical procedure. Baseline and follow-up visit will be carried out by trained personnel only. Information on all participants will be under a pseudonym. Prior to the study informed consent will be obtained from all participants.

In case of death of a study participant between baseline and follow-up visit this information will be retrieved from the insurance company, his/her general practitioner or the register office. 


\section{Cross-sectional design (additional measurements at baseline)}

Of the 2000 study participants in whom GFR was estimated using the conventional MDRD-equation a subsample of 600 subjects will be defined with oversampling of subjects under risk for CKD based on MDRD-GFR. The participation in the longitudinal part is therefore a prerequisite for the participation in the cross-sectional study part. In this subset of 600 participants kidney function will be assessed by measuring plasma clearance with the exogenous filtration marker lohexol.

lohexol is a non-ionic, non-radioactive low osmolar x-ray contrast medium which is eliminated from plasma mainly by glomerular filtration with extra-renal elimination equalling that of Inulin. It was selected for GFR measurement because it is safe and has been used in x-ray investigations for millions of patients all over the world. Its clearance from plasma has been used for measuring kidney function for more than 20 years. In Sweden, where data has been published of a 15 year experience of its use involving more than 8000 patients, no significant adverse effects have been reported (19). By contrast urinary clearance of Inulin, the gold standard, would require extensive technical assistance, the use of a bladder catheter and a difficult chemical assay. Alternatively exogenously administered radionuclides such as ${ }^{51} \mathrm{CR}$ EDTA, ${ }^{99 \mathrm{~m}}$ TC-DTPA, and ${ }^{125}$-lothalamate have been used to measure GFR. Major drawbacks of using radio-isotopes are associated with complicated procedures for handling, storage and disposal of contaminated wastes. GFR-measurement with nonradioactive lothalamate has been applied frequently but also needs urinary clearance measurement requiring a much more complicated protocol probably difficult to fulfil in elderly participants. One shortcoming about the lohexol plasma clearance method that should be mentioned, is the fact that in patients with a GFR $<25 \mathrm{ml} / \mathrm{min} / 1.73 \mathrm{~m}^{2}$ BSA, the final slope is so flat that it cannot be reliably measured (20). 
Participants will be instructed to abstain from caffeine, paracetamol, smoking and high protein foods for 6 hours prior to and during the hospital visit. Weight and height will be recorded. Baseline venous blood samples will be taken for measurement of hematocrit and serum creatinine. A bolus of $5 \mathrm{ml}$ of $300 \mathrm{mg} / \mathrm{ml}$ lohexol will be administered via an intravenous cannula. Venous blood samples will be collected from the contralateral arm by trained study nurses approximately 2, 3 and 4 hours later and the precise times will be recorded. Participants with a known allergy to iodine or a thyroid stimulating hormone $<0.3 \mathrm{mU} / \mathrm{l}$ will not be allowed to participate in the cross-sectional part.

\section{Plasma Clearance measurement}

GFR will be calculated from plasma clearance following a bolus intravenous injection of lohexol, with the clearance computed from the amount of the marker administered and the area under the curve of plasma concentration versus time. The decline in serum levels is secondary to the immediate disappearance of the marker from the plasma into its volume of distribution (fast component) and to renal excretion (slow component). This is best estimated using a two compartment model which requires early (usually 2 to 3 time points until 60 minutes) and late ( 1 to 3 time-points from 120 minutes onward) blood sampling. Advantages include low expense, wide availability, stability in biologic fluids, and rare adverse reactions when given as a small dose ( 5 ml $300 \mathrm{mg} / \mathrm{ml}$ iodine when assayed with a sensitive HPLC assay) (21), (22), (23), (24).

The creatinine-assay will be calibrated with the creatinine-assay of the Cleveland Clinic Laboratory, which is the original MDRD-Laboratory (25). The necessity of a correct calibration of the creatinine assay has been stated repeatedly (26), (27). 


\section{Participant recruitment}

The aim is to enrol a community based sample of 2000 participants. In Berlin the insurance company called "Berliner Allgemeine Ortskrankenkasse" (AOK) gives insurance coverage to almost $50 \%$ of the persons $\geq 70$ years of age. Of approximately 370.000 (28) $\geq 70$ year olds residing in Berlin 166.500 have AOK insurance.

To avoid selection bias the AOK will randomly pick a sample of participants fulfilling the inclusion/exclusion criteria named below (table 1). From another ongoing population study of elderly persons (subproject of autonomy despite multimorbidity in old age (AMA) consortium, funded by Federal Ministry of Education and Research, http://www.ama-consortium.de/f-omaha.html) it is known that overall response rates are expected to lie between $10-20 \%$ only displaying decreasing response rates with increasing age. Thus stratified oversampling for higher ages will be performed. The AOK will contact between 10.000 and 20.000 persons. In a fractionated manner invitation letters will be sent to the potential participants offering them a free kidney check-up at one of the 13 study sites. The participants are allowed to pick the study site that is most convenient for them to reach. Study subjects will be enrolled at one of the 13 study sites. Informed consent will be obtained from every participant before enrolment.

Every participant will be informed about his/her laboratory results per mail. We think out of ethical reasons we are obliged to give a recommendation to have all elevated creatinine values or pathologic urine-results re-checked and evaluated for causes. Depending on serum creatinine values the following statements will be given on the reports based on the "Deutsche Hochdruckliga" (German Hypertension League):

Creatinine (male / female): $\leq 1.17 \mathrm{mg} / \mathrm{dl} / \leq 0.95 \mathrm{mg} / \mathrm{dl}$ : normal (laboratory threshold) 
Creatinine (male / female): $>1.17-1.5 \mathrm{mg} / \mathrm{dl} />0.95-1.5 \mathrm{mg} / \mathrm{dl}:$ moderate kidney disease

Creatinine $>1.5 \mathrm{mg} / \mathrm{dl}$ : manifest kidney disease

\section{Oversampling}

The aim of our sampling plan is to obtain equal sizes of age/sex strata defined by 5 year intervals. In the entire population the number of subjects declines with increasing age. Thus in our random sample we give higher weights to the elderly to obtain equal numbers. Moreover, as we expect less response among the elderly, weights are increased by a second factor based on the experience with a community based study in the same city that is an ongoing project of one of our institutions (Dpt. Biostatistics and Clinical Epidemiology) with the Robert Koch Institute of Germany which is the main institution of health reporting in Germany. These weights are fixed and thus back-calculation of frequencies and their standard errors will be possible.

\section{Recruitment of the subset}

600 out of 2000 participants will be asked to participate in an additional kidney function assessment determined by a gold standard method measuring plasma clearance with the exogenous filtration marker lohexol. This will take place at all three Charité University Hospital study locations with the participant again being able to choose the location.

There will be a second step of oversampling with higher weights for subjects with risk for CKD. This will be based on percentiles of the preliminary determination of kidney function using the MDRD equation. 


\section{Sample size}

1) Estimation of kidney function:

We expect a prevalence of CKD of $15 \%$ in our population. This assumption is based on data from the Third National Health and Nutrition Examination Survey (NHANES III) which is the most comprehensive source of epidemiologic data regarding CKD(29). NHANES estimated that $25.22 \%$ of men $>70$ years of age and $8.25 \%$ of women $>70$ years have a serum creatinine $\geq 1.5 \mathrm{mg} / \mathrm{dl}$. To obtain a two-sided confidence limit of $2 \%$ we need a sample size of 1225 evaluable cases. As the number of drop outs is difficult to estimate and the sample size has to be sufficient also for the longitudinal part of the study we will recruit 2000 subjects for the total sample.

(2) Construction and validation of the new estimating formula for kidney function:

We need two subsamples, one sample for development (= learning sample) and one sample for validation of the new formula. Sample size estimation refers to the validation sample. The sample in which the formula will be developed should be of the same size.

The plan is to start with the same variables that were also used in the MDRDequation. Alternative models will be built including further variables such as diabetes, hypertension, and variables linked to muscle mass (BMI, WHR), as well as laboratory variables such as urea, urine- or serum-albumin.

Precision of the new formula will be quantified according to square of the Pearson correlation. We expect a value of $81 \%$, which corresponds to $r=0.9$. We want to estimate two-sided $95 \%$ limits of confidence for this precision of $5 \%$. Thus a sample 
of evaluable subjects of $n=214$ is necessary. To adjust for dropouts of $25 \%$, the validation sample should consist of $n=285$ subjects, we want to recruit 300 subjects.

The development sample will be of the same size, thus we will have to determine GFR in 600 participants.

(3) Estimation of incidences and determination of risk factors:

In the longitudinal analysis - taking into account $25 \%$ of dropouts with respect to the initial 2000 subjects - we expect $n=1500$ evaluable subjects. We have poor prior knowledge about the observed number of events during the follow-up of two years. Depending on the size of observed rates we expect a precision of between $0.8 \%$ and $2.3 \%$ (two-sided $95 \%$ confidence limits for rates of $2 \%$ to $30 \%$ ). Accordingly we can detect hazard ratios of between 2.8 (30 events) and 1.3 (456 events) (level of significance $=0.05$ two-sided, $80 \%$ power in a significance test, prevalence of risk factors $050 \%$ ). For very rare risk factors, the detectable hazard ratios will be smaller.

\section{Statistical analysis}

(1) Construction and validation of the new estimating formula for kidney function:

Methods similar to those used by Levey et al (12) will be applied in the subsample of 600 subjects undergoing lohexol measurements. Learning and validation samples will be used to obtain unbiased estimates for the precision of the formula.

(2) Estimation of kidney function:

The formula developed in the subsample of $n=600$ subjects will be used to estimate the kidney function in the total sample of $n=2000$ subjects. This analysis will be done adjusted for the oversampling of elderly subjects. We do not expect a severe 
bias from including the 600 subjects used for the development of the formula in the overall estimation of kidney function.

(3) Estimation of incidences and determination of risk factors:

Cumulative incidences of new CKD, dialysis, and heart failure will be determined based on the follow-up after 2 years using standard calculating cumulative incidences for competing risks. Simple and multiple Cox regression will be used to obtain hazard ratios including $95 \%$ limits of Confidence for the relevant events based on the followup data. Finally, applying variable selection techniques a multivariate risk index will be developed.

All analysis will be done using the newest releases of standard software (SPSS for Windows, R).

\section{Quality assurance}

Data entry will be web based via computers with internet access at the sites of the participants' examination. For data entry an AJAX (Asynchrone Java-Script and XML) -based 21 CFR 11 conform data management system (SAPPHIRE; MEDEORA $\mathrm{GmbH}, \mathrm{Köln}$, Germany) will be used. The system possesses an encoded datamanagement, -transfer, and an audit trail as well as the classical standard components for clinical studies such as support for monitoring and query-handling. The participants' data will be transferred in an encoded fashion and with the help of an ISO 25237 conform pseudonymisation- service pseudonymized (conversion of the participant identifier [PID] to a pseudonym [PSN]). Data will be stored in a central data base. An independent center (University of Wuerzburg) has been commissioned for monitoring and quality assurance. This center has been featured with an own 
administrative access to the central database and several automated analyses and plausibility checks. Figure 2 clarifies data flow and quality assurance.

From the central database a core data set will be transferred to a core database once a year. This core data base is in custodial custody of the „KfH-foundation of preventive medicine". Data from several projects converge in this joint data base and are available for future research projects.

Individual data will be controlled using ranges during data entry. A continuous monitoring of data referring to completeness, plausibility, observer effects and distributional characteristics will be implemented by the biostatistical center. These analyses will not change the design or the hypotheses of our study, thus no adjustment for interim analysis will be necessary.

\section{DISCUSSION}

CKD is one of the large and growing public health problems that aging populations will have to face to an increasing degree. This increase in CKD may not only be agerelated but also attributed to the increasing incidences of diseases associated with kidney disease, including diabetes mellitus, hypertension and other cardiovascular diseases. Clinical assessment of kidney function to define the CKD stage is essential when practicing medicine but difficult in the elderly. The level of GFR and its magnitude of change over time are crucial to the detection of CKD and for making decisions about diagnosis, prognosis and treatment. None of the existing equations to date to estimate GFR are validated in this age group since elderly people tend to be generally underrepresented (13) and they tend to underestimate GFR. The newest development, the CKD-EPI equation arose from cross-sectional pooled data 
sets of 26 studies (12). Even though a very big sample of several thousands of participants it again included only few elderly persons.

By applying lohexol plasma clearance, which should be unbiased by urinary clearance of the marker substance and thus is a practical method in an aging population we will develop a new equation from original data exclusively for persons $\geq 70$ years of age. Apart from developing a new estimation equation to accomplish more exact numbers of prevalence also co-morbidities and risk factors being associated with or contributing to the progression of CKD in the elderly will be assessed longitudinally.

The BIS has potential limitations that should be mentioned. They include the problem of a presumably low response rate especially at that age-group leading to a certain degree of selection bias despite contacting the participants randomly. The fact that the recruitment includes participants from one insurance company only may also introduce selection bias although this company is one of the biggest insurance companies and insures more than half of the target audience covering people with different socioeconomic status. Besides we are aware of the problem that there could be a higher response rate in those patients who have not yet renal insufficiency but for some reasons are aware of a potential risk. On the other hand there might be a lower response rate in subjects with manifest renal insufficiency. A second shortcoming we will have to face will be losses to follow-up, especially in this rather old population. Starting at the age of 70 years and above we expect a presumably higher mortality rate compared to a younger cohort. In this context the rather short follow-up of two years regarding evaluating the progression of CKD was chosen. While this period may be viewed as insufficient, previous studies have demonstrated that this duration is adequate to determine progression in kidney function $(30,31)$. A 
third potential limitation would be the fact that not every participant selected for lohexol measurement will agree to participate. In this case we would replace subjects who participate in the baseline examination but refuse measurement with lohexol by subjects matched for age, sex, and kidney function according to our routine measurement. Last, in case of a newly diagnosed kidney disease we will have to face the possibility of influencing the course of disease by recommending check-ups and even treatment between baseline and follow up visit.

A validated equation estimating GFR will have implications concerning referral to the nephrologist, pharmacological treatment and dosing of potentially nephrotoxic drugs and preparing for invasive diagnostic or therapeutic procedures. Furthermore it may help to increase the pool of much needed potential living kidney donors for a growing population of elderly patients with end stage renal disease. Longitudinal assessment of incident cases of kidney function, as well as progression of CKD, co-morbidities and associations with risk factors will hopefully answer questions of the aging-kidney phenomenon.

The BIS seeks to contribute to fill these gaps by combining a cross-sectional and longitudinal approach. 


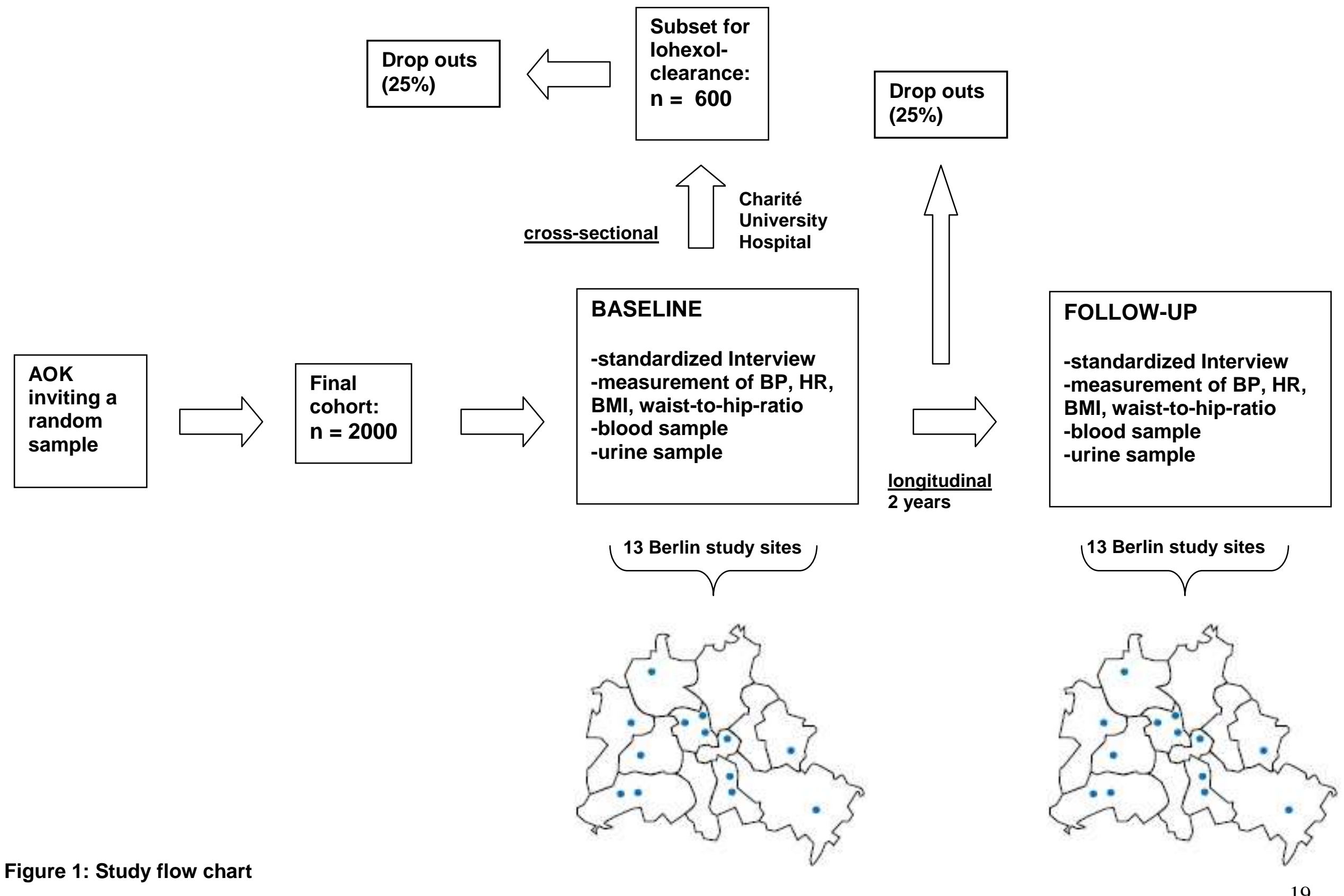




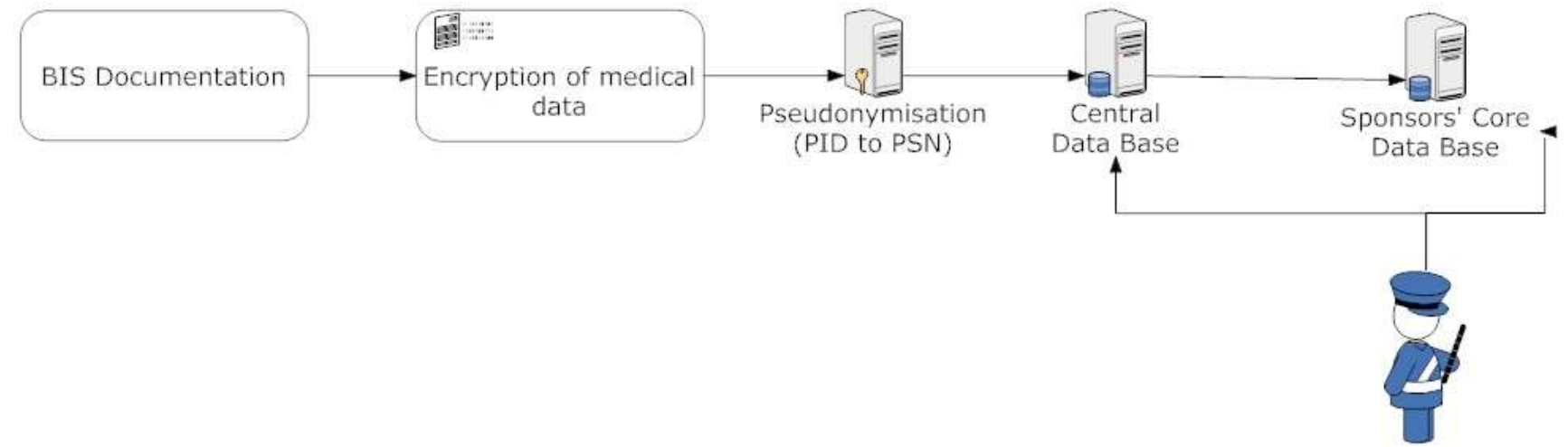

Monitoring and Quality assurance

Figure 2: Data processing and management 
Legends

Table 1: inclusion/exclusion criteria longitudinal analysis

Inclusion criteria:

- age $\geq 70$ years

- AOK insurance status

- informed consent

\section{Exclusion criteria:}

- dialysis treatment

- planned or current kidney transplantation 


\section{ACKNOWLEDGEMENTS}

The authors thank Andrej Woehrmann for his assistance in the quality assurance process and the manuscript preparation.

Grant support: This work was supported by the KfH Foundation of Preventive Medicine (www.kfh-stiftung-praeventivmedizin.de), a non-profit organisation supporting science and research in the field of prevention. Address: KfH-Stiftung Praeventivmedizin, Martin-Behaim-Strasse 20, $63263 \mathrm{Neu}$ Isenburg

Conflict of interest: none declared 


\section{REFERENCES}

1. Bundesamt S. Pressemitteilung 2006.

2. Lindeman RD, Goldman R. Anatomic and physiologic age changes in the kidney. Exp Gerontol 1986;21:379-406.

3. Goyal VK. Changes with age in the human kidney. Exp Gerontol 1982;17:321-31.

4. Abrass CK, Adcox MJ, Raugi GJ. Aging-associated changes in renal extracellular matrix. Am J Pathol 1995;146:742-52.

5. Melk A, Ramassar V, Helms LM, et al. Telomere shortening in kidneys with age. J Am Soc Nephrol 2000;11:444-53.

6. Danziger RS, Tobin JD, Becker LC, Lakatta EE, Fleg JL. The age-associated decline in glomerular filtration in healthy normotensive volunteers. Lack of relationship to cardiovascular performance. J Am Geriatr Soc 1990;38:1127-32.

7. Levey AS, Bosch JP, Lewis JB, Greene T, Rogers N, Roth D. A more accurate method to estimate glomerular filtration rate from serum creatinine: a new prediction equation. Modification of Diet in Renal Disease Study Group. Ann Intern Med 1999;130:461-70.

8. Cockcroft DW, Gault MH. Prediction of creatinine clearance from serum creatinine. Nephron 1976;16:31-41.

9. Lin J, Knight EL, Hogan ML, Singh AK. A comparison of prediction equations for estimating glomerular filtration rate in adults without kidney disease. J Am Soc Nephrol 2003;14:2573-80.

10. Rule AD, Gussak HM, Pond GR, et al. Measured and estimated GFR in healthy potential kidney donors. Am J Kidney Dis 2004;43:112-9.

11. Vervoort G, Willems HL, Wetzels JF. Assessment of glomerular filtration rate in healthy subjects and normoalbuminuric diabetic patients: validity of a new (MDRD) prediction equation. Nephrol Dial Transplant 2002;17:1909-13.

12. Levey AS, Stevens LA, Schmid $\mathrm{CH}$, et al. A new equation to estimate glomerular filtration rate. Ann Intern Med 2009;150:604-12.

13. Rule AD, Larson TS, Bergstralh EJ, Slezak JM, Jacobsen SJ, Cosio FG. Using serum creatinine to estimate glomerular filtration rate: accuracy in good health and in chronic kidney disease. Ann Intern Med 2004;141:929-37.

14. National Kidney Foundation KDG. GUIDELINE 1. DEFINITION AND STAGES OF CHRONIC KIDNEY DISEASE. 2002.

15. Coresh J, Astor BC, Greene T, Eknoyan G, Levey AS. Prevalence of chronic kidney disease and decreased kidney function in the adult US population: Third National Health and Nutrition Examination Survey. Am J Kidney Dis 2003;41:1-12.

16. Baggio B, Budakovic A, Perissinotto E, et al. Atherosclerotic risk factors and renal function in the elderly: the role of hyperfibrinogenaemia and smoking. Results from the Italian Longitudinal Study on Ageing (ILSA). Nephrol Dial Transplant 2005;20:114-23.

17. Wetzels JF, Kiemeney LA, Swinkels DW, Willems HL, Heijer M. Age- and genderspecific reference values of estimated GFR in Caucasians: The Nijmegen Biomedical Study. Kidney Int 2007;72:632-7.

18. Halbesma N, Kuiken DS, Brantsma AH, et al. Macroalbuminuria is a better risk marker than low estimated GFR to identify individuals at risk for accelerated GFR loss in population screening. J Am Soc Nephrol 2006;17:2582-90.

19. Nilsson-Ehle P. Iohexol clearance for the determination of glomerular filtration rate: 15 years' experience in clinical practice. eJIFCC 2002;13. 
20. Aurell M. Accurate and feasible measurements of GFR--is the iohexol clearance the answer? Nephrol Dial Transplant 1994;9:1222-4.

21. Brown SC, O'Reilly PH. Iohexol clearance for the determination of glomerular filtration rate in clinical practice: evidence for a new gold standard. $\mathrm{J}$ Urol 1991;146:675-9.

22. Gaspari F, Perico N, Matalone M, et al. Precision of plasma clearance of iohexol for estimation of GFR in patients with renal disease. J Am Soc Nephrol 1998;9:310-3.

23. Arvidsson A, Hedman A. Plasma and renal clearance of iohexol--a study on the reproducibility of a method for the glomerular filtration rate. Scand J Clin Lab Invest 1990;50:757-61.

24. Krutzen E, Back SE, Nilsson-Ehle I, Nilsson-Ehle P. Plasma clearance of a new contrast agent, iohexol: a method for the assessment of glomerular filtration rate. J Lab Clin Med 1984;104:955-61.

25. Murthy K, Stevens LA, Stark PC, Levey AS. Variation in the serum creatinine assay calibration: a practical application to glomerular filtration rate estimation. Kidney Int 2005;68:1884-7.

26. Coresh J, Astor BC, McQuillan G, et al. Calibration and random variation of the serum creatinine assay as critical elements of using equations to estimate glomerular filtration rate. Am J Kidney Dis 2002;39:920-9.

27. Hallan S, Asberg A, Lindberg M, Johnsen H. Validation of the Modification of Diet in Renal Disease formula for estimating GFR with special emphasis on calibration of the serum creatinine assay. Am J Kidney Dis 2004;44:84-93.

28. Bundesamt S. Berlin i-Punkt. Dezember 2005.

29. Jones CA, McQuillan GM, Kusek JW, et al. Serum creatinine levels in the US population: third National Health and Nutrition Examination Survey. Am J Kidney Dis 1998;32:992-9.

30. Hemmelgarn BR, Zhang J, Manns BJ, et al. Progression of kidney dysfunction in the community-dwelling elderly. Kidney Int 2006;69:2155-61.

31. Levey AS, Gassman JJ, Hall PM, Walker WG. Assessing the progression of renal disease in clinical studies: effects of duration of follow-up and regression to the mean. Modification of Diet in Renal Disease (MDRD) Study Group. J Am Soc Nephrol 1991;1:1087-94. 